\title{
Stress-Responses of Performance and Microbial Community in Anaerobic Digestion System Under Long-Term Enrichment of Phenanthrene
}

\author{
Yongsen $\mathrm{SHI}^{\mathrm{a}}$, Chunli XU ${ }^{\mathrm{b}}$, Jingyi $\mathrm{LI}^{\mathrm{a}}$, Yilin $\mathrm{YAO}^{\mathrm{a}}$ and Qigui NIU ${ }^{\mathrm{a}, 1}$ \\ ${ }^{a}$ School of Environmental Science and Engineering, China-America CRC for \\ Environment \& Health, Shandong University, Qingdao 266237, Shandong, China \\ ${ }^{\mathrm{b}}$ Shandong Provincial Geo-mineral Engineering Exploration Institute, Jinan, \\ Shandong, China
}

\begin{abstract}
The expanded granular sludge blanket reactor (EGSB) was operated for 198 days to study the long-term effects of phenanthrene (PHE) enrichment on system performance and microbial community. The results showed that the PHE was significantly enriched in the reactor. The final PHE concentration in effluent and sludge reached to $1.764 \pm 0.05 \mathrm{mg} / \mathrm{L}$ and $12.52 \pm 0.42 \mathrm{mg} / \mathrm{gTS}$, respectively. While the average daily methane production was decreased by $5.0 \%-9.8 \%$ under long-term PHE exposure. The 3D-EEM of effluent indicated that PHE stimulated the microbial metabolism with the higher intensity of soluble microbial byproductlike materials (SMP) and proteins. Moreover, the removal efficiency of soluble chemical oxygen demand (SCOD) and NH4+-N gradually diminished with the enrichment of PHE. PHE shaped the microbial community, and the predominant fermentative bacteria (Mesotoga) was severely inhibited. Contrarily, the bacteria (Syntrophorhabdus, Acinetobacter, Desulfovibrio, Desulfomicrobium) involved in PHE-degradation was enriched at end of Phase V. In addition, the relative abundance (RA) of hydrotrophic methanogens (Methanofastidiosum, Methanolinea, Methanobacterium, Methanomassiliicoccus) increased by 0.96 -fold with the longterm enrichment of PHE, while the RA of acetoclastic Methanosaeta obviously decreased.
\end{abstract}

Keywords. Expanded granular sludge blanket reactor, phenanthrene, anaerobic digestion system

\section{Introduction}

In recent years, soil contamination caused by the production and transportation of petroleum has attracted increasing attentions. The polycyclic aromatic hydrocarbons (PAHs) are a common class of pollutants at contaminated sites, which has harmful effects on living and non-living taxa due to their recalcitrant and lipophilic nature [1]. Therefore, researchers attached great importance to the studies about PAHs removal [2, 3].

${ }^{1}$ Corresponding Author, Qigui NIU, School of Environmental Science and Engineering, China-America CRC for Environment \& Health, Shandong University, Qingdao 266237, Shandong, China; Email: niuqg@sdu.edu.cn. 
The biological treatment with the advantages of environmental friendliness, low operational and investment costs has been applied to remove PAHs. Many studies have focused on the aerobic biodegradation of PAHs, and the high removal efficiency has been achieved [4]. However, in the deep soil, the oxygen transfer is limited, which is not conducive to the survival of aerobic microorganisms. Thus, the anaerobic microorganisms play an important role in the attenuation of PAHs. Nevertheless, the anaerobic biodegradation of PAHs still suffers many challenges such as long biodegradation period and high microbial sensitivity [2]. So far, few studies focused on the response of system performance and microbial community in anaerobic environment under the long-term enrichment of PAHs.

In present study, the phenanthrene (PHE) was selected as the model pollutant to investigate the effects of long-term PAH exposure on the anaerobic system. Meanwhile, the carbohydrate (starch) was added to provide the sufficient carbon source for the metabolism of anaerobic microbes. The variations of biomethane production, SCOD, $\mathrm{NH}_{4}-\mathrm{N}$ and dissolved organic matter (DOM) in effluent were analyzed. Also, the succession of microbial community under the long-term enrichment of PHE was evaluated.

\section{Materials and Methods}

\subsection{Experimental Design}

The PAH of PHE was purchased from the reagent company. The inoculum sludge was taken from an industrial plant which was operated at mesophilic condition in Shandong province. The experiment was conducted in an expanded granular sludge blanket reactor (EGSB) with $6.0 \mathrm{~L}$ work volume filled with $2.96 \mathrm{~kg}$ sludge. The EGSB was operated with a HRT of $48 \mathrm{~h}$ under $35 \pm 2^{\circ} \mathrm{C}$, and the up-flow rate was constant $\left(0.69 \mathrm{~L} \mathrm{~h}^{-1}\right)$ with effluent recirculation. The characteristics of influents in different phases are shown in table 1 .

Table 1. Characteristics of influents in different phases.

\begin{tabular}{lllllll}
\hline Phases & Duration (in days) & $\mathrm{COD}(\mathrm{g} / \mathrm{L})$ & $\mathrm{NH}_{4}{ }^{+}-\mathrm{N}(\mathrm{mg} / \mathrm{L})$ & $\mathrm{C} / \mathrm{N}$ & $\mathrm{pH}$ & $\mathrm{PHE}(\mathrm{mg} / \mathrm{L})$ \\
\hline I & $0-8$ & 3 & 57.6 & 15 & $7.6 \pm 0.3$ & 0 \\
II & $9-80$ & 6 & 115.2 & 15 & $7.6 \pm 0.3$ & 0 \\
III & $81-122$ & 6 & 115.2 & 15 & $7.6 \pm 0.3$ & 1 \\
IV & $123-156$ & 6 & 115.2 & 15 & $7.6 \pm 0.3$ & 10 \\
V & $157-198$ & 6 & 115.2 & 15 & $7.6 \pm 0.3$ & 100 \\
\hline
\end{tabular}

\subsection{Analytical Methods}

\subsubsection{Chemical Analysis}

The indexes including SCOD, $\mathrm{NH}_{4}{ }^{+}-\mathrm{N}, \mathrm{pH}$, TS and VS were determined according to standard methods [5]. The volumes of biogas and biomethane were measured by a glass injector, and the $\mathrm{CH}_{4}$ content in biogas was measured via absorbing the $\mathrm{CO}_{2}$ by saturated sodium hydroxide solution. The concentration of PHE in effluent and sludge was analysed by High Performance Liquid Chromatography (Shimadzu LC-2030) [1]. 


\subsubsection{EEM Analysis}

Excitation-emission matrix (EEM) fluorescence spectrum of effluent was determined by a fluorescence spectrophotometer (Hitachi Japan, F-4600). The emission wavelengths from 200 to $550 \mathrm{~nm}$ at $5 \mathrm{~nm}$ increments and the excitation wavelengths from 200 to 500 $\mathrm{nm}$ at $5 \mathrm{~nm}$ increments were set. Milli-Q water was used as reference to eliminate the inner filter effect [6].

\subsubsection{DNA Extraction and Sequencing}

The sludge samples were washed with phosphate buffer saline (PBS) three times and centrifuged at $10000 \mathrm{G}$ for $2 \mathrm{~min}$. Microbial DNA was directly extracted from $2.0 \mathrm{~g}$ sludge of each sample with a MetaVx ${ }^{\mathrm{TM}}$ (GENEWIZ, Inc., South Plainfield, NJ, USA) according to manufacturer's instructions. Then, the full length of $16 \mathrm{~S}$ rRNA was amplified using the primers (27F: 5'-AGAGTTTGATCCTGGCTCAG-3'; 1492R: 5'GGTTACCTTGTTACGACTT-3') and sequenced using PacBio Sequel system (Pacific Biosciences, USA) at Biomarker Technologies Co, Ltd. (Beijing, China).

\section{Results and Discussion}

\subsection{PHE Impacts on EGSB Treatment Performance}

The EGSB was operated for 198 days to evaluate the variations of treatment performance with the PHE enrichment. As shown in figures $1 \mathrm{a}$ and $1 \mathrm{~b}$, the concentration of PHE in reactor gradually increased at the end of different phases after Phase II. The highest final PHE concentration in effluent and sludge reached to $1.764 \pm 0.05 \mathrm{mg} / \mathrm{L}$ and $12.52 \pm 0.42$ $\mathrm{mg} / \mathrm{g}$ TS at Phase V (influent $\mathrm{PHE}=100 \mathrm{mg} / \mathrm{L}$ ), respectively, indicating that the most of PHE was absorbed in sludge due to the hydrophobic property. The enrichment of PHE adversely affected the system performance. At Phase III (influent $\mathrm{PHE}=1 \mathrm{mg} / \mathrm{L}$ ), the average daily biomethane yield (DMY) was decreased from $1461.24 \pm 151.40 \mathrm{~mL} / \mathrm{d}$ in Phase II (influent PHE=0) to $1317.74 \pm 171.77 \mathrm{~mL} / \mathrm{d}$ (figure 1c). It is suggested that the inhibition of biomethanation was caused by feeding PHE to anaerobic system. The previous study has reported that the PHE is toxic to methanogens which have low growth rate and are sensitive to changes in the environment [1]. Interestingly, the biomethane production of reactor didn't continue to decrease after Phase III, and the average DMY of Phase IV (influent PHE $=10 \mathrm{mg} / \mathrm{L}$ ) and Phase $\mathrm{V}$ with the values of $1351.21 \pm 131.33$ $\mathrm{mL} / \mathrm{d}$ and $1388.44 \pm 106.79 \mathrm{~mL} / \mathrm{d}$ were slightly higher than Phase III. It was might ascribed to that the microbial community in reactor changed under the long-term PHE exposure, as discussed in section 3.3. However, they were still lower than that of Phase II, indicating that the biomethane production was suppressed by the PHE enrichment.

Furthermore, the long-term enrichment of PHE also posed negative effects on the removal of SCOD and $\mathrm{NH}_{4}{ }^{+}-\mathrm{N}$ (figures $1 \mathrm{~d}$ and $1 \mathrm{f}$ ). After Phase I, the average influent COD concentration was $6.0 \mathrm{~g} / \mathrm{L}$ during the whole experimental period. At Phase II (Startup period) without adding PHE, the highest removal efficiency of SCOD was achieved in Phase II with the value of $65.5 \% \pm 2.8 \%$. However, when the PHE target pollutant of 1 $\mathrm{mg} / \mathrm{L}$ was added in Phase III, the microbes in the reactor were sensitive to PHE, thus the SCOD removal efficiency decreased to $62.5 \% \pm 2.8 \%$. With increasing the influent PHE concentration, the removal efficiency of SCOD gradually decreased, and the lowest SCOD removal efficiency was found in Phase V $(55.0 \% \pm 3.1 \%)$. It is might because the 
PHE is toxic to anaerobes, which may inhibit the activities of anaerobes. Similar to the removal of SCOD, the $\mathrm{NH}_{4}{ }^{+}-\mathrm{N}$ removal efficiency continued to decline with the enrichment of PHE. Compared with Phase II $(37.3 \% \pm 8.2 \%)$, the removal efficiency of $\mathrm{NH}_{4}{ }^{+}-\mathrm{N}$ was significantly diminished by $62.7 \%$, indicating that the PHE could adversely affect bio-metabolic activity of $\mathrm{NH}_{4}{ }^{+}-\mathrm{N}$.

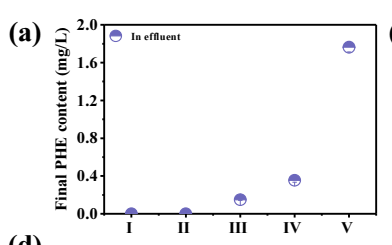

(d)

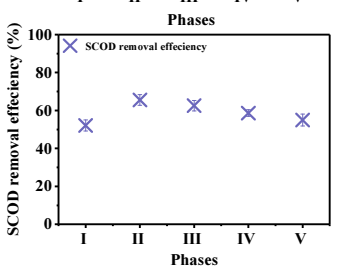

(b)

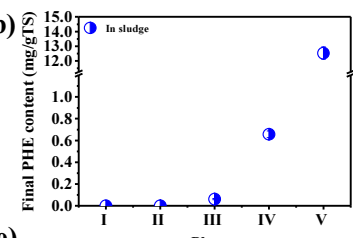

(e)

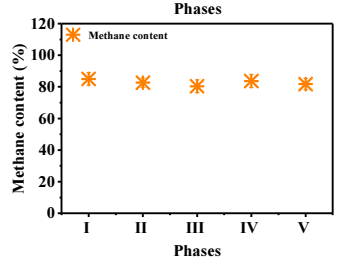

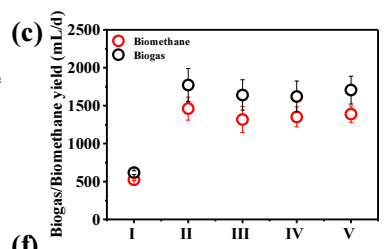

(f)

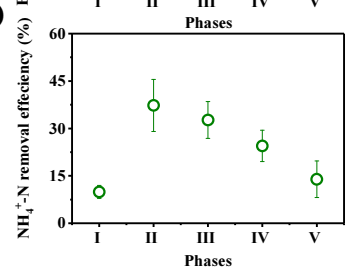

Figure 1. The variation of EGSB treatment under the long-term enrichment of PHE.

\subsection{DOM Characteristics of Effluent in Different Phases}

Three-dimensional excitation-emission matrix (3D-EEM) fluorescence spectroscopy of effluent in different phases was obtained, as shown in figure 2 . The information from the EEM could provide a high value of reference for the metabolism of microorganisms during anaerobic digestion process [7], as it usually showed the relevant characteristics of dissolved organic matter (DOM) in effluent samples comprehensively, such as the components and source of organics.

(a)

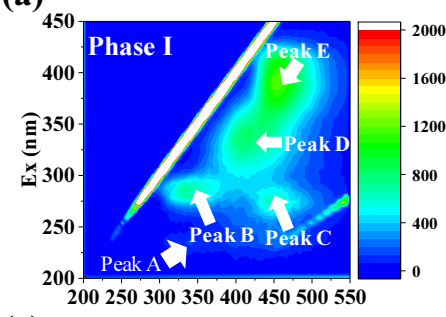

(c)

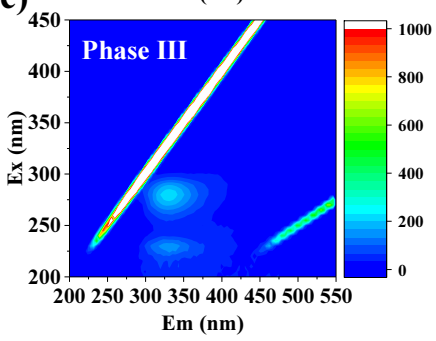

(b)

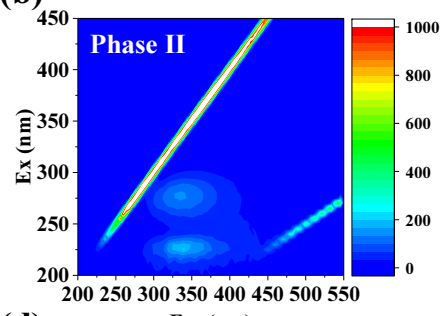

(d)

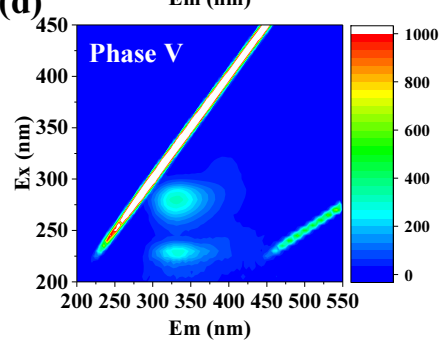

Figure 2. 3D-EEM analysis of DOM in effluents of different phases. 
The locations of the DOM fluorescence peaks were identified based on excitation/emission (Ex/Em) (figure 2a), which can be summarized into five peaks, as followed: Peak A: $E x / E m=275-285 / 325-350 \mathrm{~nm}$; Peak B: $E x / E m=225-240 / 320-340 \mathrm{~nm}$; Peak C: Ex/Em=275-280/445-450 nm; Peak D: Ex/Em=320-340/410-430 nm; Peak E: $E x / E m=380-400 / 450-470 \mathrm{~nm}$. Figure 2 showed that the characteristics of DOM in effluents of different phases. The five peaks were all found in the Phase I with the high intensity, but the peak $C$, peak $D$ and peak E belonged to humic substances [6]. It is indicated that the severe humification was occurred and the system of reactor was unstable. Fortunately, figure $2 \mathrm{~b}$ showed that only peak A and peak B were detected in the fluorescence spectrum of Phase II, and they represented soluble microbial byproductlike materials and the component of tryptophan-like protein, respectively [7], indicating that the community structure of microorganisms in reactor achieved a stable state. After Phase II, with the increase of PHE concentration, the fluorescence intensity (FI) of SMP and tryptophan-like protein gradually increased. It is obvious that FI of components in effluent of Phase V was higher than that of Phase II. The possible reason was that the PHE stimulated the bio-metabolism, which leaded to more byproducts from microbial activities including the PHE biodegradation byproducts.

\subsection{Succession of Microbial Community}

Sludge samples were collected at the end of Phase II and Phase V to identify the structure of microbial community, as shown in figure 3. Eight kinds of bacterial phyla were detected including: Thermotogae, Proteobacteria, Firmicutes, Bacteroidetes, Chloroflexi, Synergistetes, Verrucomicrobia, Actinobacteria. As the most predominant phylum, the Thermotogae occupied $87.0 \%$ in Phase II. Previous study has reported that members of Thermotogae could ferment a various of simple sugars (e.g., glucose) and complex polysaccharides (e.g., xylan and starch) [8]. However, it's relative abundance (RA) significantly reduced to $47.9 \%$ in Phase V with the enrichment of PHE, indicating the PHE posed negative effects on fermentation of substrate (starch). The RA of Proteobacteria in Phase V (39.3\%) was comparatively higher than Phase II (9.3\%). And the phyla of Firmicutes was enriched by 4.3 times in Phase V compared with Phase II (1.3\%). Lee et al. reported that Proteobacteria and Firmicutes were the dominant phyla in the oil contaminated sediment and potentially participated in the degradation of PAHs [9]. At genus level (figure 3a), eight dominant genera were found, as followed: Mesotoga, Syntrophorhabdus, Acinetobacter, Clostridium, Bacteroides, Chryseomicrobium, Desulfovibrio, Desulfomicrobium. Among them, the hydrolytic Mesotoga is the most predominant genus with the RA of $91.2 \%$ in Phase II, but it declined to $58.4 \%$ in Phase V. Clostridium and Bacteroides were responsible for carbohydrate hydrolysis in anaerobic system [10]. Their RA increased from $1.3 \%$ and $0.5 \%$ in Phase II to $7.3 \%$ and $2.9 \%$ in Phase V, respectively. Moreover, the abundance of Syntrophorhabdus and Acinetobacter which were typical acetogens increased with the enrichment of PHE in Phase V, reaching to $11.8 \%$ and $10.8 \%$, separately. Compared with Phase II, Desulfovibrio and Desulfomicrobium belonging to sulfate-reducing bacteria exhibited higher abundance, which are reported to take part in the anaerobic degradation of PHE [11]. Above results indicated that the enrichment of PHE shaped the bacterial community.

The variations of archaeal community are showed in figure $3 \mathrm{c}$. It was obvious that acetoclastic Methanosaeta occupied the highest proportion with the value of $71.1 \%$ in the Phase II. However, it's abundance was decreased by $29.8 \%$ in Phase V, indicating that the increase of PHE caused suppression on the growth of Methanosaeta. Conversely, 
the RA of hydrotropic methanogens including Methanofastidiosum, Methanolinea, Methanobacterium, and Methanomassiliicoccus was promoted from $28.3 \%$ in Phase II to $55.6 \%$ in Phase V. It was suggested that the long-term enrichment of PHE affected methanogenic activities of archaea.

(a)

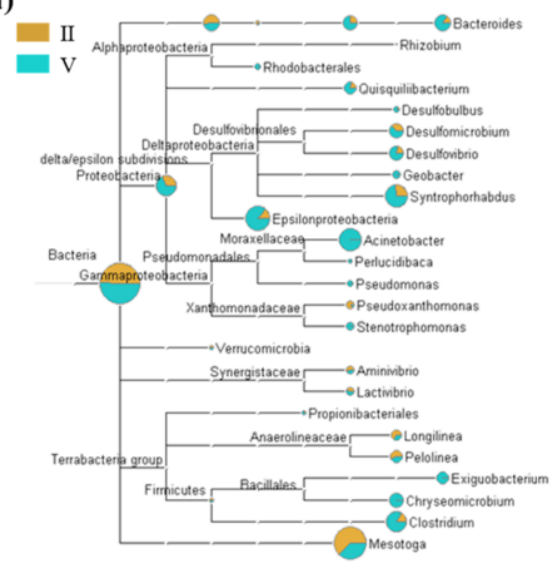

(b)

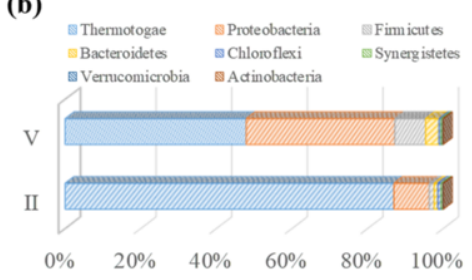

(c)

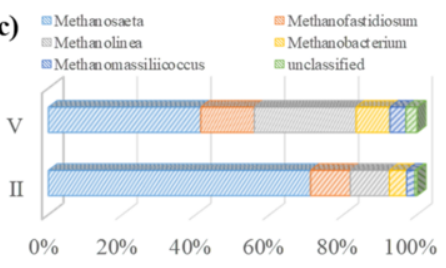

Figure 3. (a) The relative abundance (RA) of bacteria at different levels; (b) the RA of bacteria at phylum level; (c) the RA of methanogenic archaea at genus level.

\section{Conclusions}

The variations of anaerobic digestion performance and microbial community were investigated in EGSB under the long-term enrichment of PHE. There was slight suppression on biomethane production after the addition of PHE. Compared with Phase II (influent $\mathrm{PHE}=0$ ), the average daily biomethane yield was diminished by $5.8-9.8 \%$ in the next phases. Meanwhile, PHE posed negative effects on the removal of SCOD and $\mathrm{NH}_{4}{ }^{+}-\mathrm{N}$, and the biggest inhibition ratio $\left(16.0 \%\right.$ of SCOD removal and $62.7 \%$ of $\mathrm{NH}_{4}{ }^{+}-$ $\mathrm{N}$ removal) in Phase $\mathrm{V}$ (influent $\mathrm{PHE}=100 \mathrm{mg} / \mathrm{L}$ ). The sequencing results showed that the abundance of predominant fermentative bacteria (Mesotoga) significantly decreased under PHE exposure. Conversely, the typical acetogens (Syntrophorhabdus, Acinetobacter) and sulfate-reducing bacteria (Desulfovibrio, Desulfomicrobium) which potentially participated in biodegradation of PHE were enriched with increasing PHE concentration. Moreover, PHE also affected the structure of archaeal community. The growth of hydrotropic methanogens (Methanofastidiosum, Methanolinea, Methanobacterium, and Methanomassiliicoccus) were promoted, while acetoclastic Methanosaeta was inhibited.

\section{Acknowledgements}

The authors' research is supported by the National Natural Science Foundation of China (51608304 and U1806216) and Young Scholars Program of Shandong University (2018WLJH53). China Postdoctoral Science Foundation (2017M622209 and 2019T120599) was also acknowledged. 


\section{References}

[1] Lin C, Wu P, Liu Y, Wong J W, Yong X, Wu X, Xie X, Jia H and Zhou J 2019 Enhanced biogas production and biodegradation of phenanthrene in wastewater sludge treated anaerobic digestion reactors fitted with a bioelectrode system Chemical Engineering Journal 365 1-9.

[2] Bonaglia S, Broman E, Brindefalk B, Hedlund E, Hjorth T, Rolff C, Nascimento F J A, Udekwu K and Gunnarsson J S 2020 Activated carbon stimulates microbial diversity and PAH biodegradation under anaerobic conditions in oil-polluted sediments Chemosphere 248126023.

[3] Feng L, Chen J, Wang F, Chen Y and Luo J 2019 Acidogenic fermentation facilitates anaerobic biodegradation of polycyclic aromatic hydrocarbons in waste activated sludge ACS Sustainable Chemistry \& Engineering 7 5404-5411.

[4] Sponza D T and Gok O 2010 Effect of rhamnolipid on the aerobic removal of polyaromatic hydrocarbons (PAHs) and COD components from petrochemical wastewater Bioresource Technology 101 914-924.

[5] TSEP Administration 2002 The water and wastewater monitoring analysis method editorial board Water and Wastewater Monitoring Analysis Method (Beijing: China Environmental Science Press).

[6] Li D, Song L, Fang H, Li P, Teng Y, Li Y Y, Liu R and Niu Q 2019 Accelerated bio-methane production rate in thermophilic digestion of cardboard with appropriate biochar: Dose-response kinetic assays, hybrid synergistic mechanism, and microbial networks analysis Bioresource Technology 290121782.

[7] Song L, Song Y, Li D, Liu R and Niu Q 2019 The auto fluorescence characteristics, specific activity, and microbial community structure in batch tests of mono-chicken manure digestion Waste Management $\mathbf{8 3}$ 57-67.

[8] Serna-Garcia R, Zamorano-Lopez N, Seco A and Bouzas A 2020 Co-digestion of harvested microalgae and primary sludge in a mesophilic anaerobic membrane bioreactor (AnMBR): Methane potential and microbial diversity Bioresource Technology 298122521.

[9] Lee D W, Lee H, Lee A H, Kwon B O, Khim J S, Yim U H, Kim B S and Kim J J 2018 Microbial community composition and PAHs removal potential of indigenous bacteria in oil contaminated sediment of Taean coast, Korea Environmental Pollution 234 503-512.

[10] Wang H, Qu Y, Li D, Ambuchi J J, He W, Zhou X, Liu J and Feng Y 2016 Cascade degradation of organic matters in brewery wastewater using a continuous stirred microbial electrochemical reactor and analysis of microbial communities Scientific Reports 627023.

[11] Shi K, Liang B, Guo Q, Zhao Y, Sharif H M A, Li Z, Chen E and Wang A 2021 Accelerated bioremediation of a complexly contaminated river sediment through ZVI-electrode combined stimulation Journal of Hazardous Materials 413125392. 